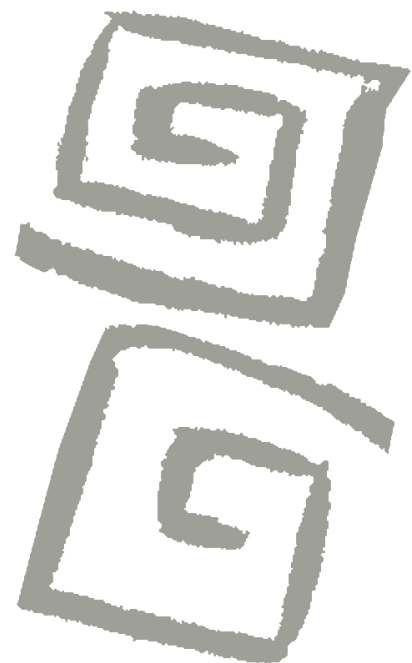

\title{
El poder médico y la crisis de los vínculos de confianza en la medicina contemporánea
}

\author{
Medical power and the crisis in bonds of trust within \\ contemporary medicine
}

Yuri Nishijima Azeredo', Lilia Blima Schraiber ${ }^{2}$

'Psicólogo. Estudiante de Maestría, Programa de Posgrado en Medicina Preventiva, Faculdade de Medicina, Universidade de São Paulo (USP). Becario, Coordenação de Aperfeiçoamento de Pessoal de Nivel Superior (CAPES), Brasil. yuri.azeredo@gmail.com

${ }^{2}$ Médica, Doctora en Medicina. Profesora, Departamento de Medicina Preventiva, Faculdade de Medicina, Universidade de São Paulo (USP). Investigadora $1 \mathrm{~A}$, Conselho Nacional de Desenvolvimento Científico e Tecnológico (CNPq), Brasil. liliabli@usp.br
RESUMEN Basados en el contexto brasileño, en este artículo abordamos el poder médico en términos de los actuales conflictos en las relaciones intersubjetivas que el médico establece en su trabajo, conflictos considerados aquí como producto de una crisis de confianza vinculada a los recientes cambios históricos de la práctica médica. Al interpretar esos conflictos como cuestiones de orden ético y moral, recurrimos a las formulaciones teóricas de Hannah Arendt para analizar con mayor profundidad dicha crisis de confianza. De este modo, a partir de los conceptos arendtianos de "crisis", "tradición", "poder", "autoridad" y "natalidad", realizamos una lectura con nuevos significados de estos conflictos, que posibiliten futuros caminos y nuevas soluciones que eviten una nostalgia del pasado.

PALABRAS ClAVES Poder Profesional; Humanización de la Atención; Ética Médica; Humanidades.

ABSTRACT Based on the Brazilian context, this paper addresses medical power in terms of the current conflicts in the intersubjective relationships that doctors establish in their work, conflicts considered here as a product of a crisis of trust connected to recent historical transformations in the medical practice. Reading these conflicts as questions of an ethical and moral order, we use Hanna Arendt's theoretical formulations to further analyze this crisis of trust. In this way, utilizing the concepts of "crisis," "tradition," "power," "authority," and "natality," we search for new meanings regarding these conflicts, enabling new paths and solutions that avoid nostalgia for the past.

KEY WORDS Power, Professional; Humanization of Assistance; Ethics, Medical; Humanities. 


\section{INTRODUCCIÓN}

En este trabajo abordaremos los cambios de la relación médico-paciente en la práctica médica contemporánea. A través del contraste entre la medicina establecida por la "tradición", concepto que tomamos de Arendt ${ }^{(1)}$, y la medicina tecnológica actual ${ }^{(2)}$, analizamos de qué modo las transformaciones en las relaciones del trabajo y la inserción de nuevas tecnologías afectan un elemento central del encuentro clínico. Se trata de un vínculo de confianza entre el médico y el paciente construido a lo largo del desarrollo de la medicina liberal, durante el siglo XIX y hasta mediados del siglo XX. Ese vínculo se encuentra extremadamente tensionado en la contemporaneidad, debido a las rupturas en la interacción por las que hoy pasan esas relaciones.

La presente reflexión forma parte de una investigación titulada "Salud Colectiva y Filosofía: contribuciones de Hannah Arendt al debate de la humanización" ${ }^{\text {[a] }}$, que estudia las producciones bibliográficas brasileñas en el campo de la salud colectiva sobre el tema de la humanización/deshumanización en salud. Estas producciones señalan la ocurrencia progresiva, en los servicios de salud, de maltratos, agresiones verbales, negligencia en los cuidados, u obstáculos en el acceso y en el uso de los servicios, que son vistos como actos de violencia contra los usuarios.

Aunque mucho se discute, por un lado, sobre las condiciones de trabajo de los profesionales y las dificultades del sistema de salud brasileño para brindar un mejor modelo de atención y, por otro, acerca de la base extremadamente técnica de la formación y de la práctica profesional, un aspecto que, a nuestro juicio, debe ocupar el centro del debate es la transformación del poder médico en violencia institucional.

Entendemos que dicha transformación requiere una reflexión crítica más profunda, por lo que recurrimos al pensamiento de Hannah Arendt, cuya diferenciación entre los conceptos de poder, autoridad y violencia se ha revelado como fuente fundamental para este análisis.

La elección del pensamiento de esta autora se ve reforzada por nuestra tesis: comprendemos la mencionada crisis de los vínculos de confianza como producto de diversas rupturas en las interacciones entre los médicos y otros sujetos presentes en la práctica médica. Dicha crisis estaría relacionada con conflictos de orden ético y moral en la práctica de los médicos, que conducen a la pérdida de autoridad legítima de esos profesionales en las intervenciones sobre el cuerpo y la persona del paciente. Debido a esto, buscamos en Arendt, como gran pensadora de aquellas cuestiones contemporáneas de la acción política que involucran autoridad y moral, algunas formulaciones teóricas que apoyen el desarrollo de esa tesis.

\section{EL MÉDICO Y EL PODER}

Encontramos en la sociología médica y en la sociología de las profesiones el fundamento macrosocial para el fenómeno contemporáneo de la crisis de los vínculos de confianza. La sociología es un campo de estudio privilegiado para la reflexión sobre la relación entre la agencia y la estructura: la relación entre la acción humana y la determinación estructural de las relaciones sociales a través de las denominadas "relaciones de poder". De acuerdo con Turner ${ }^{(3)}$, cuando la sociología trata el área médica, lo que le interesa es la relación entre el saber y el poder en la distribución de la salud y la enfermedad dentro del cuerpo social. Se trataría de una sociología preocupada por el papel que tienen los discursos médicos en la fundamentación del poder médico que, por su parte, diseña las posibilidades de comprensión de la relación entre salud y enfermedad, así como su distribución.

Este autor señala que, para abordar el poder médico, fue fundamental la clasificación de la imprecisa noción de enfermedad en tres categorías distintas: disease, illness y sickness. De forma general, podemos decir que la primera alude al carácter anatomopatológico de 
los desórdenes, como indica Foucault ${ }^{(4)}$; la segunda categoría se refiere a la experiencia subjetiva de enfermarse; y la última (sickness) designa el sentido de la enfermedad relacionada con las fuerzas culturales macrosociales y culturales, en cuanto a la determinación de los papeles de los enfermos y de las enfermedades dentro de la sociedad. Así, este abordaje muestra la enfermedad más allá del carácter biológico, colocándolo en el constructo social de las sociedades modernas.

Turner menciona, además, que otro punto a tener en cuenta es la división del trabajo en el área de la salud, ya que los médicos se responsabilizan del tratamiento de las patologías (disease), los psicoterapeutas se ocupan de los padecimientos (illness), y los cientistas sociales buscan comprender el proceso de la enfermedad socialmente construida (sickness). El autor señala que el estatuto de cientificidad de los conocimientos de esas profesiones son jerárquicos, puesto que la intervención médica es vista socialmente como precisa, exacta y científica mientras que la interpretación del cientista social, habitualmente, es considerada como una opinión.

Sin embargo, si Turner identificó los distintos poderes relacionados con el ejercicio de la autoridad en la intervención profesional, en función de la diversificación de los saberes implicados en esas definiciones del proceso salud-enfermedad se pueden añadir otras modalidades de saberes que también compiten en jerarquías subalternas por el estatuto de cientificidad: fruto de las respectivas experiencias con la enfermedad, tendremos el saber de los propios enfermos (que son los que efectivamente lidian con las terapéuticas para su cuidado) o saber popular, y el saber práctico de los médicos ${ }^{(2)}$.

Este último deriva de sus experiencias asistenciales y fue fundamental en la construcción de la medicina moderna al tener otro estatuto en la práctica del médico liberal frente a su casi nula importancia en la medicina tecnológica actual. De esa forma, el saber práctico de los profesionales fue relevante para la construcción de la tradición de la medicina, en términos del encuentro clínico y de la creación de vínculos de confianza.
La jerarquía diferenciada con relación al estatuto científico de esos conocimientos produce que la patología sea entendida como entidad natural y neutra sobre la cual el médico actuará a partir de técnicas científicamente fundamentadas. Por lo tanto, serían intervenciones técnicas de carácter neutro sobre una disfunción de origen natural. En esta visión, ampliamente criticada por la sociología de la salud y de la medicina, se sustenta el poder del médico, su neutralidad ante los intereses sociales y políticos y su naturalización como acción.

Es fundamental la contribución del filósofo francés Michel Foucault para la deconstrucción de la enfermedad como ente natural, así como para la formulación del saber como dispositivo de control. Especialmente relevante para nuestro estudio es la preocupación del autor por examinar la relación entre ciertos discursos médicos y el ejercicio del poder. Esos discursos evolucionan a la par del aumento de la vigilancia de los Estados a través de la disciplina del cuerpo y del control de las poblaciones. En ese sentido, Foucault ve la misma forma de actuar en la clínica, en el asilo y en la prisión, a través de una forma de vigilancia panóptica ${ }^{(5)}$. En el análisis de la relación entre el discurso, las prácticas, los grupos profesionales y el Estado, Foucault argumenta que el saber-poder se organizó sobre la disciplina del cuerpo individual, dentro de esas instituciones, y sobre el cuerpo de las poblaciones, a través del nacimiento de la medicina social ${ }^{(6)}$.

La epistemología foucaultiana sostiene que el ser humano solo puede conocer (o ver) aquello que permite el lenguaje $y$, por consiguiente, el discurso científico es comprendido como una narración determinada por las convenciones lingüísticas: diferentes sociedades en la historia de la humanidad poseen diferentes convenciones $y$, por lo tanto, realidades distintas. En el caso de la patología, deja de ser posible comprenderla como un evento natural fuera del lenguaje que lo describe, y pasa a convertirse en un ente producido por el discurso médico que refleja el modo dominante del pensamiento de la sociedad ${ }^{(4),(7)}$. Por lo tanto, para el autor, 
lo que entendemos como enfermedad es un efecto de las relaciones de poder-saber. De acuerdo con Foucault ${ }^{(4),(5)}-y$ también con lo señalado por el sociólogo americano Elliot Freidson ${ }^{(8)}$ - si la expansión del conocimiento científico incorporado por la medicina le otorgó a los médicos un prestigio y una influencia enorme a fines del siglo XIX, esto ocurrió porque todo un campo institucional -que también formó el Estado modernocreó las condiciones para ello, permitiendo a los médicos definir lo que es norma y lo que es desvío.

Al avanzar sobre cuestiones de la medicina en el siglo $\mathrm{XX}$, Freidson ${ }^{(8)}$ muestra que la constitución de una profesión médica, dotada de autonomía, que monopoliza conocimientos, prácticas, instituciones y su evaluación social, incluyó una serie de procesos. En primer lugar, la escuela médica universitaria de la Edad Media preparó el contexto en el que se desarrollaron los criterios que identificaron a ese grupo específico de trabajadores. Después, debido a la importancia que el estudio universitario tenía para la elite de la época, rápidamente los médicos graduados obtuvieron el apoyo del Estado para convertirse en árbitros de su propio trabajo.

Eso significó el control sobre la formación de los futuros médicos por parte de sus colegas, así como la limitación y hasta la prohibición de otras actividades que se ocupaban del mismo objeto. Posteriormente, el Estado cedería a los médicos el derecho de limitar, supervisar y dirigir el ejercicio de las ocupaciones que pudieran rivalizar con la medicina. Esta es la definición de autonomía profesional: el derecho de diagnosticar y prescribir según los criterios habituales del saber médico y el derecho de que los pares evalúen el propio trabajo. Desde esta perspectiva, se entiende por poder médico a la capacidad de la corporación de controlar la formación de nuevos médicos, así como de las profesiones paramédicas, además de una autonomía profesional autorregulada ${ }^{(8)}$.

Pierre Bourdieu, en la compilación de entrevistas y diálogos titulada Um convite à Sociologia Reflexiva ${ }^{(9)}$, define campo como una red o una configuración de relaciones objetivas entre posiciones. Estas posiciones pueden ser identificadas materialmente $y$ también en función de las relaciones que guardan entre sí $^{(10)}$. Los campos, así pensados, son relacionales, dinámicos, contingentes y en constante cambio, lo que apunta a la necesidad de que se piensen de forma relacional o dialéctica ${ }^{(11)}$. El dinamismo que marca las estructuras del campo no se da, sin embargo, de forma aleatoria, sino que sigue una lógica propia, que determinará su funcionamiento particular ${ }^{(9)}$. Por lo tanto, pensar en el campo médico es pensar en un espacio constituido por un conjunto de instituciones que proponen, a través de leyes y reglas, la cuestión sanitaria en una sociedad, o sea, las políticas de salud y sus formuladores, los diversos profesionales que actúan en las redes de servicios, las instancias de formación de esos profesionales, y los propios usuarios de los servicios de salud ${ }^{(10)}$.

Bourdieu formula también el concepto de habitus: "...un cuerpo estructurado, un cuerpo socializado, un cuerpo que ha incorporado las estructuras inmanentes de un mundo o de un sector particular de ese mundo"(12). Así, el estudio del poder médico a partir de la perspectiva de Bourdieu se dará en el análisis de las instituciones que, en red, son formadas por el campo médico y en las relaciones de estas con la formación del habitus profesional.

Por su parte, el sociólogo Paul Starr ${ }^{(13)}$ considera que el poder médico se funda en la autoridad técnico-científica de la profesión, a partir de la legitimidad científica de sus conocimientos y de la dependencia de la sociedad de esos mismos conocimientos. De esta forma, el poder médico se originaría en la posibilidad de que los grupos profesionales organizados generen nuevas formas de dependencia de sus conocimientos y su competencia. Lo que le da un carácter distintivo a esas relaciones de dependencia entre el público consumidor y la profesión es el hecho de que los grupos profesionales, a través de los mecanismos institucionales más diversos, consiguen que sus interpretaciones del mundo sean consideradas como verdades y que se transformen en referencias sociales 
y subjetivas que ayudan a comprender el mundo y nuestra realidad. A este tipo particular de autoridad, Starr" ${ }^{(13)}$ la denomina "autoridad cultural". En ella, si bien el médico es buscado y consultado por los consumidores, no tiene el poder de imponer un tratamiento determinado, en caso de que el paciente se rehúse a seguirlo.

¿Qué significa esa imposibilidad de los médicos de imponer algo a sus pacientes? Desde nuestra perspectiva, y de acuerdo con el estudio de Freidson ${ }^{(8)}$, la medicina es una profesión consultante $y$, como tal, los pacientes la buscan espontáneamente y lo hacen porque están aculturados en ese mismo orden social, compartiendo esa medicalización de los determinantes de la enfermedad. Ese proceso se da por la educación de las poblaciones ${ }^{(8)}$ y, más que por la eficacia técnico-científica o por el "éxito técnico", -como diría Ayres ${ }^{(14)}-$, por la conquista de la confianza en el encuentro clínico ${ }^{(15)}$.

Tanto en el éxito técnico como en el desarrollo de los vínculos de confianza -aunque con cierta asimetría de autoridad de los sujetos implicados- se fundamenta el proceso material y simbólico para la construcción de una "tradición" de la profesión médica, ideario en el que los profesionales y la sociedad se aculturan. Un amplio conjunto de valores y virtudes corresponde a esa "tradición", como se discutirá más adelante. Sucede que parte de ese ideario persiste hasta hoy, sobre todo aquel referido a la autoridad cultural, incluso con diversos valores y virtudes presentes en la época de su construcción y actualmente en contraste con los profundos cambios de conformación material, institucional y técnica de la profesión.

De hecho, como muestran los relatos de médicos paulistas, entrevistados como testigos de los cambios históricos de la profesión médica en $\mathrm{Brasil}^{(2)}$, con el desarrollo de una medicina progresivamente tecnológica, los vínculos que fundaron la confianza en el médico fueron rompiéndose, ocasionando, en la actualidad, importantes crisis en el encuentro clínico con respecto a las relaciones intersubjetivas y las tensiones radicales para que el médico pueda ejercer su poder.
Deesta forma, identificamos la "tradición" con la modalidad liberal de la práctica y de la profesión en medicina ${ }^{(2),(15)}$. El profesional se sitúa en el mundo laboral como detentor de los medios de producción de su trabajo y como regulador del flujo de su clientela. Esta posición, configurada en Brasil a lo largo de las primeras tres décadas del siglo $\mathrm{XX}$, hace referencia a una autonomía de carácter mercantil, debido a la amplia libertad en la ejecución de su práctica como producción social de un servicio, y libertad en la fijación de su remuneración, a la que se suma la autonomía técnica y la de la organización y control de su servicio ${ }^{(2)}$.

Donnangelo ${ }^{(16)}$ definirá tal condición como "autonomía típica", en contraste con las readaptaciones que sufre la inserción del médico en su mercado de trabajo en las décadas de 1970 y 1980. Con la rápida incorporación de las tecnologías a mediados del siglo $X X$, sea en forma de nuevos tratamientos o como recursos diagnósticos, casi desaparece el trabajo médico liberal que, además, se trataba de una "práctica solitaria"(2),(8), es decir, basada en un único productor. El médico, entonces, creaba e incorporaba la cultura de trabajar solo y valerse, sobre todo, de su autoridad en las decisiones clínicas que tomaba.

La imposibilidad del productor individual de hacerse cargo de los costos de adquisición y mantenimiento de los medios de producción de su servicio llevan la categoría médica rumbo al asalariamiento, tanto en el sector público como en el sector privado, con el surgimiento de las grandes empresas de servicios de salud.

Algunos autores consideran que esa nueva posición de los médicos forma parte de un proceso mayor de desprofesionalización ${ }^{(17)}$ o de proletarización ${ }^{(18),(19)}$ de los médicos, aspectos que no serán abordados en este texto. Lo que destacamos es el impacto y el significado que generó esa nueva posición en los vínculos relacionales de los médicos y los pacientes. Esto se debe a que los médicos pierden el control sobre el acceso del público, lo que los convierte, para la población, en intermediarios en el acceso a la salud: el paciente busca al médico solo si este integra 
su plan de salud o si está ligado a determinado hospital. El médico tiene contacto con el paciente solo en el hospital o si se "encuentra" en la cartilla del plan de salud ${ }^{(2)}$.

A ese "anonimato", que evidencia la despersonalización de la relación médico-paciente, se suman las exigencias de la productividad empresarial, pública o privada, además de la valorización progresiva de una práctica centrada en el uso de los exámenes complementarios o de las tecnologías terapéuticas. Todo ello genera la percepción, tanto por parte de los médicos como de los pacientes, de que la esfera de la interrelación está muy mal. A todo ello hay que añadir el crecimiento de las tecnologías de la información y su incorporación en la práctica médica, lo que también contribuye al desgarro de los vínculos interpersonales en los que reposa la confianza.

Dos declaraciones de médicos entrevistados en el referido estudio de Schraiber ${ }^{(2)}$ ilustran esas cuestiones:

Había una época en que las personas elegían mucho a los médicos por una cuestión de confianza en un buen médico, y sería muy difícil que se fuera de ese de confianza, perdiera la confianza, y le preguntara a otro, y regresara a él para operarse. El sistema de confianza ha quedado un poco... debilitado [...] Por ejemplo, yo no podía atender aquel día por algún imprevisto y las personas pedían ser atendidas por otro médico, que pudiera atenderlas ese día. Entonces, en vez de ir al médico $X$, va al médico $Y$, y punto. No cambia nada. Es ir al médico, y basta. Da igual uno $u$ otro... ${ }^{(2 \text { p. 123-124) }}$

Hoy en día, una cosa que ha cambiado es el acceso a la información. El paciente te llega con la información... jllega ya con la carpetita debajo del brazo, y discute de igual a igual! [...] ¿Cómo me posiciono como médico, con relación a todo esto? Porque yo voy a usar valores para la decisión que son diferentes de los del hijo de un paciente con cáncer. ¡Para él eso que está ahí va a ser siempre una maravilla! ${ }^{(2 \mathrm{p} .200-201)}$

\section{TÉCNICA, VALORES Y RUPTURAS EN LA INTERACCIÓN}

La práctica médica es el ejercicio de una determinada técnica de intervención, una terapéutica que busca la curación o el control de enfermedades. Aunque se haya dicho mucho sobre el carácter ético de esa técnica-entendido por el sentido común como una consecuencia de la realización de esa intervención sobre las personas- lo que afirmamos aquí es la consustancialidad de la ética con la técnica, debido al entrelazamiento completo de esas dimensiones en la práctica del médico. A tal punto se da ese entrelazado que la propia técnica puede ser considerada "moral dependiente"(2),(15), una acción revestida de valores que, por medio de las modalidades de interacción, permiten establecer el uso más objetivo de los conocimientos de carácter científico y de sus recursos tecnológicos. En este sentido, afirmamos que la ética no se reduce a una disposición personal del profesional, como realización individual e independiente de la acción técnica. La ausencia de ese ejercicio ético compromete la propia fundamentación de la acción científica ${ }^{(2)}$. A fin de cuentas, la práctica médica es la intervención del hombre sobre el hombre mismo, intermediada por la tecnología y por la ciencia. Si esa práctica se fundamentara solo a través de aspectos técnicos, la relación transmutaría hacia la intervención del hombre sobre un objeto. Por esta razón, no se trata solo de adicionar al acto técnico elementos éticos, como una especie de complemento circunstancial. Muchas veces vemos incentivos para estimular, por ejemplo, la conversación con el paciente, como solución a las críticas hechas en torno al tiempo irrisorio de la consulta que, a su vez, demostraría la falta de interés por los pacientes. Así, si se plantea como algo externo a la competencia y al uso de los recursos científicos que deja de interactuar con el momento propiamente tecnológico de la intervención, esa conversación corre el riesgo de establecerse desde un falso interés por el paciente o como una conversación "inútil", en contraste con aquella que genera el conocimiento necesario para la intervención ${ }^{(2)}$. 
Estas consideraciones tienen como propósito alertar sobre una visión diferente de la práctica médica en función de cómo los propios profesionales la llevan a cabo. Para estos, su intervención reside en la dimensión técnica, y las cuestiones sociales o éticas actúan como perturbadoras o alterando la libertad de la técnica ${ }^{(2)}$. Desde esta concepción, cuando existe un reconocimiento de la necesidad ética en el plano de las relaciones entre el médico y el paciente como, por ejemplo, en situaciones de conflicto con la libertad técnica, la solución pasa por agregar un comportamiento personal más amistoso o simpático con el paciente. Pero ese componente no interactúa con la técnica.

En la producción asistencial, actualmente se produce un desplazamiento de la práctica hacia el polo científico-tecnológico, con una aparente supresión de los valores éticos antes adoptados. Se trata de un gran cambio en las formas de relacionarse de los médicos con los pacientes o incluso entre los profesionales, sean médicos o no. Desde la década de 1990, con el desarrollo del modelo de la medicina tecnológica ${ }^{(2 \mathrm{p} .67)}$, las modalidades de interacción se transformaron, agravando los desafíos profesionales. A causa de la generalidad del científico frente al caso singular de la práctica asistencial, estos desafíos siempre están presentes en forma de conflictos de deberes, conflictos morales. Podemos incluso afirmar que, a raíz del propio desarrollo del polo científico-tecnológico, se amplía mucho el abanico de acciones y, en consecuencia, el abanico de conflictos. Estas son las diversas situaciones que hoy requieren atención y cuidado en los servicios de salud y que sirven de referencia para la crisis de carácter humanístico, tan debatida ${ }^{(20),(21)}$.

Según $\operatorname{Arendt}^{(1)}$, la crisis se experimenta como una ruptura de la "tradición" cuando, frente a un conflicto, el pasado ya no proporciona criterios que validen y autoricen las respuestas; es decir, no podemos valernos de las mismas referencias que han estructurado el mundo hasta ahora. Siendo así, los médicos no pueden apelar a los modos a través de los cuales la medicina del pasado habría solucionado los conflictos. De esta forma, la situación de crisis no se caracteriza como degeneración, sino como ruptura de las normas que distinguen, por ejemplo, lo verdadero de lo falso ${ }^{(22)}$. O sea, la pérdida de referenciales antes legítimos, en los que se constituyó la confianza, para poder resolver los conflictos en los vínculos relacionales.

Con esto queremos decir que, para nosotros, la tradición está históricamente emplazada en la constitución de la medicina de la modernidad, por tratarse de una práctica que se fundamenta en la ciencia moderna y por construir, al interior de ese mismo fundamento, vínculos de confianza entre médicos y pacientes. Así, actúa como medicina liberal, cuyo modo de realización converge más con lo artesanal que con lo tecnológico. Nos referimos aquí a la figura clásica del médico en su consultorio particular, como el pequeño productor privado de servicios que cuenta con un arsenal tecnológico considerablemente menor al actual, pero que ya procede a la intervención en los marcos de la biociencia moderna.

Lo que cambió radicalmente fueron los vínculos de confianza fundados en las relaciones intersubjetivas construidas por la medicina liberal y a los que se podía recurrir en situaciones dilemáticas. La ausencia de esos vínculos es el resultado de rupturas en la interacción, dadas por el cambio material y simbólico que la medicina tecnológica provocó en la posición de los médicos, de otros profesionales de la salud y de los pacientes, con respecto a las relaciones que establecen entre sí: el lugar que ocupan y el sentido que tienen en las relaciones de asistencia y cuidado hoy son otros, con relación a lo tradicional.

Freidson $^{(8)}$ caracteriza la práctica del médico como el difícil ejercicio de un juicio complejo y de la toma de decisiones arriesgadas, dificultades que apasionan a los que eligen la profesión, como lo demuestran las declaraciones de los estudiantes de medicina en muchos estudios ${ }^{(23)}$, y cuyo enfrentamiento no depende solo de sus competencias científicas, como apresuradamente presupone el sentido común. El mismo autor muestra que esta complejidad, como desafío profesional, reside en que, en la medicina de la modernidad, el juicio clínico tuvo que responder 
al pragmatismo de la intervención que necesitaba siempre encontrar soluciones, incluso para los casos en que la ciencia no tenía una respuesta. De ahí la identificación de la medicina como práctica artesanal en la modernidad. Sin embargo, esa falta de respuesta científica se sustentaba en la idea de que la ciencia todavía no tenía una respuesta, siendo esta una formulación derivada del gran valor dado al conocimiento científico y a los recursos tecnológicos, convertidos en "un bien en sí mismo" en la modernidad.

El pragmatismo le exigió a los médicos una doble actuación técnica: por un lado, valerse del conocimiento científico cuando fuese pertinente $y$, por otro, valerse de una sabiduría práctica propia de la profesión, es decir, la rica experiencia adquirida por cada cual y por el conjunto de los médicos sobre casos semejantes y sin fundamento científico para la acción. Fue por esta razón que la medicina se establece en la modernidad como la ciencia y el arte de curar.

Además, como afirma Schraiber ${ }^{(2)}$, al usar la ciencia como aplicación de un conocimiento universal a casos singulares, justamente porque la práctica médica se realiza en forma de consultas individuales (lo que se corresponde con la práctica liberal del consultorio privado), la complejidad del juicio clínico se intensifica. A ello se suma la necesidad de que el médico decida cómo adecuar el uso del conocimiento universal a las contingencias del caso particular, es decir, en qué medida y qué tan apropiado es este uso, dadas las particularidades específicas de la situación del caso.

Destacamos aquí, no solo el carácter reflexivo que se le exigía al juicio médico, sino también el hecho de que por esa razón el médico fue quien simbolizó a la medicina, en evidente contraste con los referenciales actuales.

\section{REFERENCIALES CONTEMPORÁNEOS}

Si no es el médico, ¿cuál es el referencial de la medicina? Podemos responder: la tecnología; los equipos o las grandes instituciones, como los hospitales que son sede de las tecnologías; o los seguros de salud y la seguridad estatal que dan acceso a las tecnologías. Las tecnologías son hoy el referencial de la medicina y la simbolizan. Por lo tanto, generan comportamientos profesionales que acentúan esa referencia enfatizando la pérdida de los antiguos referenciales.

Tal configuración produce en el ámbito intersubjetivo un cambio según el cual los medios pasan a constituir fines en sí mismos, alterando el sentido de la relación entre las personas: ya no es el médico el que se relaciona con el paciente, mientras los recursos técnicos están al servicio de esa relación. El médico ahora es un "medio" para la relación de los pacientes con la tecnología de la medicina. Del mismo modo, los pacientes se han convertido en medios para los médicos en su relación con el conocimiento - la ciencia y los descubrimientos-, y medios también para la relación del médico con los engranajes empresariales propios del ejercicio de la profesión.

Cuando médicos y pacientes son instrumentalizados en el sentido de convertirse en un medio para determinado fin, es fundamental recordar que la hegemonía del utilitarismo sistemático en la modernidad, que impone su lógica de medios y fines a todas las categorías de la vida, trae consigo importantes consecuencias. Entre ellas, la imposibilidad de "comprender la diferencia entre utilidad y pleno significado, que expresamos lingüísticamente mediante la distinción entre "con el fin de" y "en beneficio de"(24 p.191). Así, todo se coloca en la cadena de medios $y$ fines $y$, como consecuencia, todo se vuelve un medio para algún fin y solo será la razón de algo, o "en beneficio de". Esta vía posibilita que la utilidad (para qué es o para qué sirve) suprima el significado (qué es), cuya consecuencia es presentada por Arendt: "El 'con el fin de' ha pasado a ser el contenido del 'en beneficio de'; en otras palabras, la utilidad establecida como significado genera la significación"(24 p.192).

No se trata aquí de hacer apología de la abolición de los recursos tecnológicos en salud, como si nuestro horizonte fuera 
la restitución de la medicina tradicional. En este sentido, concordamos con Ayres ${ }^{(25)}$ en la conceptualización del cuidado como el acto asistencial que amplía y flexibiliza la normatividad de la aplicación de las tecnociencias; normatividad que surge de la constitución del cuerpo como ente abstracto y genérico en las ciencias, en sus productos tecnológicos y en los protocolos de los procedimientos. Ampliación y flexibilización significan aquí no solamente tratar el cuerpo y las enfermedades en sus singularidades morfofuncionales, sino también, junto al paciente, establecer un proyecto terapéutico compartido. Así, si el éxito técnico y el práctico ${ }^{(14)}$ dialogaran a través de la permeabilidad entre los aspectos técnicos y los no técnicos, la relación entre el médico y el paciente dejaría de ser el encuentro del médico con la ciencia intermediado por un cuerpo y se convertiría en un recorrido compartido a través del diálogo intersubjetivo, cuya dirección es el resultado de esa negociación entre los sujetos y sus proyectos de vida pretendidos y posibles ${ }^{(14)}$.

Médicos brasileños, entrevistados a fines de la década de $1990^{(2)}$ y en los primeros años de la década siguiente ${ }^{(21)}$, expresaron un gran malestar por los nuevos contextos del ejercicio profesional. Manifestaron, con cierta indignación, cómo se convirtieron en anónimos para los pacientes, pero sin percibir que hicieron lo mismo con sus pacientes.

Otro aspecto que ilustra estas transformaciones de la práctica médica se puede observar en el modo de utilizar los exámenes complementarios a la historia clínica, a los que denominan solo como exámenes diagnósticos, por lo que desaparece la noción de que complementan el raciocinio clínico ${ }^{(21)}$.

\section{DESAFÍOS CONTEMPORÁNEOS}

¿Qué hacer en situaciones clínicas en las que los exámenes más usuales no muestran un diagnóstico taxativo? ¿Dar más crédito al raciocinio clínico, valiéndose de la reflexión y de la experiencia adquirida, manteniendo la tecnología como complemento, o creer más en la ayuda de la tecnología? ¿Seguir investigando e intentar conocer más, o aceptar la posibilidad de proponer una terapéutica que requiera ser revisada? ¿Aceptar que la investigación genera desventajas en cuanto a los costos, lo que puede apartar clientes, o mantener la tecnología de punta como criterio, elitizando al público?

Estas cuestiones fueron señaladas por muchos de los médicos entrevistados. Son situaciones de conflicto que le marcan a la medicina contemporánea qué camino tomar en el juicio clínico, cuya solución podría ser el encuentro de la mejor resolución posible para el caso en su contexto, es decir que en la elección entre dos valores igualmente aceptados (valor de raciocinio clínico y valor de la objetividad de los exámenes) se opte cada vez por el mejor camino. La mejor manera no es siempre la tecnología o la experiencia práctica de la profesión, sino que cada opción es, al mismo tiempo, una elección responsable y prudente ante una situación particular y concreta del caso. Responsabilidad y prudencia caracterizan la deliberación moral y el ejercicio ético de la intervención técnico-científica.

Sin embargo, ya sabemos que la tendencia general de los profesionales es la de aumentar la espiral de los exámenes, ya que están situados en un referencial tecnológico, creándose un círculo vicioso en que los excesos producen más excesos y los límites de la tecnología son los más difíciles de ver.

Sobre esta cuestión del aumento en la utilización de los Ilamados exámenes complementarios, Hannah Arendt nos ayuda a analizar los cambios en el proceso productivo en la modernidad. Para la autora, uno de los signos de la revolución industrial es la transformación de toda la producción humana en bienes de consumo:

La interminabilidad de la producción solo puede asegurarse si sus productos pierden su carácter de uso y cada vez se hacen más objetos de consumo, o bien si, para decirlo de otro modo, la proporción de uso queda tan tremendamente acelerada que la objetiva diferencia entre uso y consumo, entre la relativa duración 
de los objetos de uso y el rápido ir y venir de los bienes de consumo, disminuye hasta la insignificancia. ${ }^{(24}$ p.137)

El aumento en la utilización de los exámenes complementarios muestra cómo estos se convierten en bienes consumibles, incluso se constituyen en una de las razones económicas de la existencia de las empresas médicas y del complejo médico-industrial. En la actualidad, la relación del paciente con la salud se construye sobre el consumo de los exámenes diagnósticos y en la posibilidad de acceso a los aparatos hospitalarios y, en esa relación, el médico ocupa el lugar de mero intermediario. Ello también se debe a la transformación del significado y del ejercicio relativo a la práctica responsable. Los referentes para esta última también cambiaron, como veremos más adelante, pero ahora analizaremos otro calificador/atributo de la práctica médica: el de ser un acto difícil que implica además tomar decisiones que siempre son arriesgadas.

Las decisiones clínicas implican riesgos basados en incertidumbres, porque refieren a conocimientos de naturaleza probabilística sobre eventos vitales y, por ello, con fluctuaciones según el resultado esperado. Pero más allá de estas incertidumbres, hay otras propias de los estratos sociales y subjetivos que son consustanciales a las características naturales de esos eventos, complicando aún más nuestro tema. Si bien puede parecer que la decisión clínica se refiere a las enfermedades, de hecho la intervención se realiza sobre los enfermos: el desarrollo de la decisión terapéutica concreta depende del todo bio-psico-social que el enfermo representa. Es interesante la reflexión de Freidson ${ }^{(8)}$ acerca del siguiente contraste: la práctica médica está impregnada de incertidumbre, pero lo que puebla el imaginario social es la referencia a la intervención segura, porque está científicamente fundamentada. La construcción del conocimiento científico en salud se fundamenta en el establecimiento de universales sobre el funcionamiento del cuerpo, de la mente y de las enfermedades ( $d i-$ seases). El lugar de las decisiones médicas se encuentra en el espacio entre lo universal de la ciencia y la singularidad del caso atendido. La aplicación directa de los universales de la ciencia a través de sus aparatos tecnológicos y farmacéuticos es uno de los principales cambios de la medicina, desde su carácter liberal al tecnológico, y puede ser entendido como una de las razones de la percepción, entre médicos y pacientes, de que se están convirtiendo en meros intermediarios de estas relaciones.

Para los profesionales también hubo, en su ideario ocupacional, una construcción de seguridad, aunque diferente de la anterior, que ha tomado esa misma dirección. Analizar ese movimiento es la clave para nuestra comprensión sobre cómo se dio la construcción de los vínculos de confianza en la relación médico-paciente durante la medicina liberal y por qué con ese cambio que sucede en la medicina tecnológica tales vínculos se rompen y se pierde la tradición de las relaciones.

En la medicina liberal tal construcción se basa en que los médicos creen en sus propios discernimientos, o sea, se creían efectivamente capaces de usar bien la articulación entre lo científico y la experiencia práctica, y se reforzaba la figura del médico como referencia de una intervención prudente y segura. $Y$ de allí que también estaban a disposición, profesionalmente, para acompañar siempre sus intervenciones, siguiendo muy de cerca sus casos $y$, ante cualquier necesidad de revisión de la terapéutica adoptada, admitían la posibilidad y la necesidad de esa revisión.

Sobre la base de esa conducta moral de la profesión, se construyó la confianza en la práctica liberal. Se asentó tal confianza en la disposición de pensar sobre el caso, al juzgarlo, y en la disposición de acompañar el caso. Esos aspectos hoy ya se han transformado, sea por recurrir casi de forma automática a la tecnología, como una especie de juicio fijo y a priori; sea por el gran cambio, en la medicina empresarial contemporánea, respecto de las posibilidades de seguimiento de los casos y de la vinculación interpersonal, dado que en la medicina 
empresarial los médicos pierden el control sobre los clientes y sobre los equipos con los que trabajan.

Según Arendt ${ }^{(24)}$, la comprobación telescópica realizada por Galileo de que la tierra es la que gira alrededor del sol y no al contrario, eleva los instrumentos creados por el hombre a una posición de tamiz de la verdad, instaurando de manera generalizada la desconfianza en los sentidos humanos con relación a su búsqueda. La desconfianza sobre el juicio médico parece crecer, en la modernidad, en proporción al desarrollo de los instrumentos que auxilian el discernimiento del profesional, pero que, en la contemporaneidad, tienden a sustituirlo.

De esta forma, el desarrollo científico tecnológico, por un lado, amplió las posibilidades de la intervención médica, generando mayor comodidad para los profesionales en su desempeño y, por otro, representó la molestia de verse reducidos a intermediarios en el acceso a las tecnologías. Comprometida la figura del médico como referencia para la buena práctica, la crisis coloca bajo sospecha la propia autoridad del profesional, creando en el médico las actitudes defensivas que vemos hoy con frecuencia y que se ejercitan también a priori. Al buscar que prevalezca una autoridad que se cree aún más legítima en razón del mayor desarrollo de los fundamentos científicos de su práctica, el médico pretende imponer su perspectiva en vez de dialogar con el paciente, asegurando tal imposición a través del control que efectivamente tiene todavía del acceso a las diversas tecnologías, diagnósticas y terapéuticas, y del acceso al propio sistema de salud. Esas actitudes refuerzan la pérdida de la interacción y se hacen presentes en relaciones en las cuales la autoridad es sustituida por la violencia, lo que lleva a los movimientos sociales a luchar por una nueva (otra) humanización de la medicina. Así, el médico, ante la pérdida de la legitimidad para hacer uso del antiguo puesto de poder que antes ocupaba, convierte la relación solo en un ejercicio de mando y control del paciente, situación en la que, como dice Arendt ${ }^{(26)}$, no hay ningún poder, solo violencia.
Como indica Gomes ${ }^{(21)}$, al discutir la enajenación de los médicos con respecto a la naturaleza social de su trabajo y su práctica técnica, observamos que al interior de la profesión hay cierto descontento y extrañeza por el hecho de que todo lo que haga ya no será percibido como orientado hacia lo humano, en esa práctica que a ellos les parece altamente "humanizada".

En este sentido, debemos considerar, y preferimos creer, que también los profesionales quieren superar la crisis de confianza. Pero es necesario tener en claro que los referentes del pasado ya no pueden operar, por lo que no hay reconquistas, sino nuevas conquistas para las cuales será necesario abrirse, como sujetos históricos, a otras y nuevas experiencias de la profesión. Sin embargo, esta no es una posición fácil, como se puede apreciar a partir de las reflexiones de Marilena Chauí ${ }^{(27)}$, sobre apelar a la ideología en situaciones de crisis: los individuos tienen una gran dificultad para percibirse como sujetos históricos precisamente por la consciencia que ello despierta con relación al cambio de nuestras posiciones en la sociedad. Chauí comenta este aspecto al analizar cómo, especialmente para la producción científica y para sus representantes, en los límites de una ideología de la armonía y de la permanencia de las posiciones sociales, el término "crisis" se transforma en la noción de "desajuste", englobando como solución, en los marcos de esa misma ideología, un retorno a la posición supuestamente de "ajuste" construida anteriormente (al desajuste/crisis).

La tentación de las reconquistas es grande pero, como dice Arendt ${ }^{(1)}$, es necesario nacer de nuevo, en el sentido histórico-social. Nacer de nuevo como sujetos, renovando la tradición. En ese sentido, solo para estimular nuevas reflexiones, señalamos dos dimensiones, en las que podemos buscar este nuevo nacimiento como sujetos, o una nueva "natalidad", en las referencias arendtianas. La primera dimensión está en la apuesta a la "sociedad de derechos", tal como la queramos construir, y en la cual se puedan establecer nuevas relaciones y otra forma de autoridad. La segunda, que 
interactúa con la anterior, reside en el hecho histórico de que el médico liberal se construyó como sujeto y autoridad con base en su práctica de consultorio, en la que podemos identificar la marca de una práctica "solitaria", situación con la que el médico identificó además su autonomía profesional. Así, los cambios en las relaciones de trabajo expuestas en este artículo, sumados a la diversidad de profesionales que componen el área de la salud actualmente, requieren reflexiones que renueven (y no rescaten) sus posiciones en el mundo del trabajo. Por ello, compartir juicios y decisiones con otros sujetos-pacientes o miembros del equipo de trabajo, y renovar la forma de actuar responsable de los profesionales, puede ser un buen reinicio.

\section{NOTAS FINALES}

a. El proyecto de investigación fue aprobado por el Comité de Ética en Investigación (código No. 728479), con el Certificado de Apresentação para Apreciação Ética (CAAE) No. 33405514.0.0000.0065.

\section{AGRADECIMIENTOS}

Este artículo recibió el apoyo de la Coordenação de Aperfeiçoamento de Pessoal de Nivel Superior (CAPES), a través de la beca de posgrado 2015, y del Conselho Nacional de Desenvolvimento Científico e Tecnológico $(\mathrm{CNPq})$, a través de la beca de investigación No. 306881/2013-6

\section{REFERENCIAS BIBLIOGRÁFICAS}

1. Arendt H. Entre o passado e o futuro. 7a ed. São Paulo: Perspectiva; 2011.

2. Schraiber LB. O médico e suas interações: a crise dos vínculos de confiança. São Paulo: Hucitec; 2008.

3. Turner BS. Medical power and social knowledge. 2a ed. London: Sage Publications; 1995.

4. Foucault M. O nascimento da clínica. 7a ed. São Paulo: Forense Universitária; 2011.

5. Foucault M. Vigiar e punir: nascimento da prisão. 42a ed. Petrópolis: Vozes; 2015.

6. Foucault M. O nascimento da medicina social. En: Microfísica do poder. 28a ed. Rio de Janeiro: Graal, 2014.

7. Foucault M. A ordem do discurso. 19a ed. São Paulo: Edições Loyola; 2009.
8. Freidson E. Profissão médica: um estudo de sociologia do conhecimento aplicado. São Paulo: Editora UNESP, Sindicato dos médicos; 2009.

9. Bourdieu P, Wacquant L. Um convite à sociologia reflexiva. Rio de Janeiro: Relume-Dumará; 2002.

10. Castro R. Génesis y práctica del habitus médico autoritario en México. Revista Mexicana de Sociología. 2014;76(2):167-197.

11. Everett J. Organizational research and the praxeology of Pierre Bourdieu. Organizational Research Methods. 2002;5(1):56-80.

12. Bourdieu P. Razões práticas: sobre a teoria da ação. 9a ed. São Paulo: Papirus Editora; 2008.

13. Starr $P$. The social transformation of American medicine. New York: Basic Books; 1982.

14. Ayres JRCM. Sujeito, intersubjetividade e práticas de saúde. Ciência \& Saúde Coletiva. 2001;6(1):63-72.

15. Schraiber LB. O médico e seu trabalho: Limites da liberdade. São Paulo: Hucitec; 1993.

16. Donnangelo MCF. Medicina e sociedade. 2a ed. São Paulo: Hucitec; 2011.

17. Freidson E. Professionalism reborn: Theory, prophecy and policy. Chicago: The University of Chicago Press; 1994.

18. Larson MS. The rise of professionalism: a sociology analysis. London: University of California Press; 1977.

19. Machado MH. Os medicos e sua prática profissional: as metamorfoses de uma profissão. [Tesis de doctorado]. Rio de Janeiro: Instituto Universitário de Pesquisas do Rio de Janeiro; 1996.

20. Deslandes SF. Humanização: revisitando o conceito a partir das contribuições da sociologia médica. In: Deslandes SF, (org). Humanização dos cuidados em Saúde: 
conceitos, dilemas e práticas. Rio de Janeiro: Editora Fiocruz; 2006.

21. Gomes RM. Trabalho médico e alienação: as transformações das práticas médicas e suas implicações para os processos de humanização/desumanização do trabalho em saúde. [Tese de doutorado]. São Paulo: Faculdade de Medicina, Universidade de São Paulo; 2010.

22. Carvalho JSF. Educação: uma herança sem testamento. [Tese de Livre Docência]. São Paulo: Faculdade de Educação, Universidade de São Paulo; 2013.

23. Rios IC, Schraiber LB. Humanização e humanidades em medicina: A formação médica na cultura contemporânea. São Paulo: Editora Unesp; 2012.
24. Arendt H. A condição humana. 12a ed. Rio de Janeiro: Forense Universitária; 2014.

25. Ayres JRCM. O cuidado, os modos de ser (do) humano e as práticas de saúde. Saúde e Sociedade. 2004;13( 3): 16-29.

26. Arendt H. Sobre a violência. Rio de Janeiro: Civilização Brasileira; 2013.

27. Chauí M. Cultura e democracia: (o discurso competente e outras falas). São Paulo: Moderna; 1981.

\section{FORMA DE CITAR}

Azeredo YN, Schraiber LB. El poder médico y la crisis de los vínculos de confianza en la medicina contemporánea. Salud Colectiva. 2016;12(1):9-21.

Recibido: 6 de julio de 2015 | Versión final: 24 de septiembre de 2015 | Aprobado: 30 de noviembre de 2015

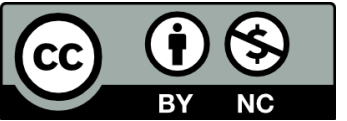

Este obra está bajo una licencia de Creative Commons Reconocimiento-NoComercial 4.0 Internacional. Reconocimiento - Permite copiar, distribuir y comunicar públicamente la obra. A cambio, se debe reconocer y citar al autor original. No Comercial - Esta obra no puede ser utilizada con finalidades comerciales, a menos que se obtenga el permiso. 